\title{
ARTICLE \\ Clinical Characteristics and Risk Factors of Atrial Fibrillation in the Elderly
}

\section{Hong Li*}

Shanghai Jincheng Nursing Hospital, Shanghai, 201100 China

\begin{tabular}{|c|c|}
\hline ARTICLE INFO & ABSTRACT \\
\hline Article history & \multirow{9}{*}{$\begin{array}{l}\text { Objective: To investigate the epidemiobgical characteristics of the elder- } \\
\text { ly with atrial fibrillation (AF) in age, gender, the types of AF and relative } \\
\text { causes. And To analyze the risk factors of persistent AF (PeAF), then use } \\
\text { them to guide to prevent and treat for AF in the elderly.Methods: Col- } \\
\text { lect the data of elderly patients with AF who were admitted to Liaoning } \\
\text { People's Hospital from September } 1,2016 \text { to September } 30,2017 \text {, and } \\
\text { summarize the epidemiological characteristics of AF in the elderly. The } \\
\text { risk factors for PeAF were analyzed by Spearman correlation analysis } \\
\text { and mltivariate logistic regression analysis. Result: } 1 \text {. In the elderly, the } \\
\text { number of AF cases increase with age. The group of } 75-84 \text { years old was } \\
\text { the largest part. The number of older females was larger than male in all } \\
\text { ages. It was important to pay more attention to old woman with AF. } 2 \text {.In } \\
\text { the elderly, nonvalvular atrial fibrillation is popular. The top three causes } \\
\text { of AF in the elderly were CHD, hypertension and heart failure. In the } \\
\text { elderly, comorbidities were frequent, and the management was a major } \\
\text { therapeutic objective. } 3 \text {.CRP, left atria diameter were the independent } \\
\text { risk factors for PeAF in the elderly. }\end{array}$} \\
\hline Received: 4 April 2019 & \\
\hline Accepted: 17 April 2019 & \\
\hline Published Online: 30 April 2019 & \\
\hline Keywords: & \\
\hline Elderly & \\
\hline Atrial fibrillation & \\
\hline Epidemiology & \\
\hline Risk factor & \\
\hline
\end{tabular}

\section{Introduction}

$\mathrm{A}$ trial fibrillation $(\mathrm{AF})$, as one of the most common tachyarrhythmias, is currently affecting 33 million people worldwide and growing at an annual rate of 5 million $^{[1]}$. It is known as the "Cardiovascular Epidemiology in the 21st Century". Some reports showed that there are about 2.7 million to 6.7 million people with AF in the United States; and it is estimated that it will increase to 5.6 million to 15.9 million people by $2050^{[2,3]}$. In 2010 , studies showed that about 8.8 million elderly people over 55 years of age in Europe suffered from AF. If the age and sex ratio remained stable, the number of people with $\mathrm{AF}$ is expected to double by $2060^{[4]}$. By 2050 , there will be 7.2 million patients with AF and 2.9 million patients with atrial fibrillation-related stroke in $\mathrm{Asia}^{\left[{ }^{[5]}\right.}$. Most of the patients with AF are the elderly, and the incidence of AF increases with age. The Framingham study showed that the incidence of $\mathrm{AF}$ increased significantly after the age of 60 , doubling on average every 10 years ${ }^{[6]}$; In 2004, Zhou et al. carried out a large-scale epidemiological study on 29079 cases of 14 natural populations. Among them, 224 patients with $\mathrm{AF}$ had a standardized prevalence rate of $0.61 \%, 0.5 \%$ in people aged $50-59$, and $7.5 \%$ in those aged over $80^{[7]}$. Many studies have shown that the incidence of AF increases with age. Most of the patients are over 60 years old. Researchers suggest that AF research should be carried out for people over 60 years old.

*Corresponding Author:

Hong $\mathrm{Li}$,

Shanghai Jincheng Nursing Hospital,No. 700 Beisong Highway, Minhang District, Shanghai, 201100 China;

E-mail: lisalihong@hotmail.com. 
In the special group of the elderly, AF often coexists with a variety of diseases, showing a comorbid state, and mutual influence, mutual cause and effect. The occurrence of AF is often associated with many organic cardiovascular diseases, such as coronary heart disease, hypertension, heart failure, valvular disease, cardiomyopathy, sick sinus syndrome, etc., and is also closely related to other systemic diseases, such as hyperthyroidism, diabetes, sleep apnea syndrome, ischemic stroke, chronic kidney disease, etc. AF not only affects the quality of life of patients, but also significantly increases the risk of heart failure and stroke ${ }^{[8]}$

With the continuous progress of the aging of the social population, the number of patients with $\mathrm{AF}$ is also increasing. $\mathrm{AF}$ and aging are unavoidable medical and social problems all over the world, which is of great significance to conduct the in-depth study on AF in the elderly. At present, the treatment of $\mathrm{AF}$ is the research focus of most scholars, radiofrequency ablation of AF, left atrial appendage occlusion, pacemaker therapy and other diverse treatments. The cost of clinical treatment is high; the risk factors of intervention in $\mathrm{AF}$ can reduce the burden of $\mathrm{AF}$ to a certain extent. The occurrence of AF varies in different races and regions. According to the AHA/ACC/HRS guidelines for AF management in 2014, approximately $35 \%$ of patients with AF are older than 80 years old. In most atrial fibrillation-related studies, the selection conditions of the subjects are relatively broad and there is no specific age limit. As a result, the average age of the study cohort is about 5 years younger than that of the population with AF. Whether these findings are applicable to the elderly is still controversial ${ }^{[9]}$. Therefore, it is necessary to carry out relevant researches on the elderly with AF. This study was conducted in a single-center retrospective analysis of 700 elderly patients with AF who were over 65 years old and hospitalized in Liaoning People's Hospital. The clinical characteristics of elderly patients with AF were summarized, including age, sex, type of AF and related etiology. The related risk factors of elderly patients with persistent $\mathrm{AF}$ were analyzed to provide direction and reference for the prevention and treatment of elderly patients with $\mathrm{AF}$.

\section{Materials and Methods}

\subsection{Research Subjects}

The medical records of elderly patients with atrial fibrillation who were admitted to the Heart Center of Liaoning People's Hospital from September 1, 2016 to September 30, 2017 were counted. Included subjects: Patients aged 65 years or older with evidences of AF such as electrocar- diogram or dynamic electrocardiogram or a clear history of AF. Excluded subjects: Patients with transient/reversible etiology. These include acute myocardial infarction, acute myocarditis, untreated hyperthyroidism and AF caused by recent electrophysiological examinations, angiography, pacemaker surgery, and cardiothoracic surgery, etc. The data of multiple hospitalized patients were based on the first hospitalization data and were counted only once.

\subsection{Methods}

\subsubsection{Clinical Data Collection}

It should be include patient age, gender, etiology and type of AF, past medical history, and related laboratory indicators, i.e., C-reactive protein (CRD), triglyceride (TG), cholesterol (TC), low-density lipoprotein (LDL), blood uric acid (BUA), serum creatinine (CREA) and so on, echocardiography recorded left atrial meridian (LAD), left ventricular ejection fractions(LVEF\%).

\subsubsection{Diagnostic Criteria for Major Diseases}

(1) Elderly: age over 65

(2) Diagnostic criteria for atrial fibrillation: A. At least one ECG or 24 hour dynamic electrocardiogram (DCG) proved the diagnosis of AF. $\quad$ B. A clear history of atrial fibrillation in the past. At least one of them should be met.

(3) Type of AF:

A. Paroxysmal atrial fibrillation: a kind of AF with a duration of $<7$ days, which can be converted within 48 hours, with self-limiting.

B. Persistent atrial fibrillation: a kind of AF with a duration of $>7$ days, which cannot be converted by itself.

C. Valvular atrial fibrillation: heart valve disease diagnosed by echocardiography, combined with AF. Heart valve diseases include rheumatic heart disease, senile degenerative valvular disease.

D. Non-valvular atrial fibrillation: exclusion of valvular heart disease by echocardiography, with other diseases such as coronary heart disease, cardiomyopathy, pulmonary heart disease, congenital heart disease, hypertension, hyperthyroidism, electrolyte imbalance, anemia, combined with AF.

E. Isolated atrial fibrillation: Clinical examination confirmed no combined heart disease such as coronary heart disease, cardiomyopathy, pulmonary heart disease, congenital heart disease, and other systemic diseases such as hypertension, hyperthyroidism, electrolyte imbalance, anemia, combined with AF.

(4) Cardiomyopathy: mainly including dilated cardiomyopathy, hypertrophic cardiomyopathy, etc. 
(5) Hypertension: according to the 2010 Guidelines for the Prevention and Treatment of Hypertension in China, patients with systolic blood pressure $(\geq 140 \mathrm{~mm} \mathrm{Hg})$ and/ or diastolic blood pressure $(\geq 90 \mathrm{~mm} \mathrm{Hg}$ ), or who have previously diagnosed hypertension, who are receiving antihypertensive therapy and whose blood pressure level is within the normal range .

(6) Diagnostic criteria for heart failure: class II and above of cardiac function were diagnosed as heart failure according to the cardiac function classification of the New York Heart Association (NYHA).

(7) Diagnostic criteria for diabetes: using the diagnostic criteria proposed by the American Diabetes Association in 2012 , i.e., fasting blood glucose $\geq 7.0 \mathrm{mmol} / \mathrm{L}$ and/or OGTT test was $\geq 11.1 \mathrm{mmol} / \mathrm{L}$. Or a definite diagnosis of diabetes in the past.

(8) Diagnostic criteria for coronary heart disease: A. previous history of angina pectoris or significant ST-T changes showed by electrocardiogram, 24-hour DCG, exercise plate test, etc., during hospitalization; B. coronary artery stenosis $(\geq 50 \%$ ) confirmed by coronary angiography; C. history of acute myocardial infarction or previous definite history of myocardial infarction. One of the above conditions should be met.

\subsubsection{Cases Grouping}

(1) 2 groups by gender: male and female.

(2) 3 groups by age: 65 to 74 years old, 75 to 84 years old, 85 years old and above.

(3) 2 groups according to the type of AF: A. Paroxysmal atrial fibrillation and persistent atrial fibrillation; B. valvular atrial fibrillation and non-valvular atrial fibrillation.

\subsubsection{Statistical Methods}

SPSS 18.0 program was used for statistical data processing; Chi-square test selected for counting data; $t$ test selected for measurement data; $\mathrm{P}<0.05$ was statistically significant. Spearman correlation analysis was used for statistical significance indicators, and logistic regression analysis was conducted for correlative indicators.

\section{Results}

\subsection{Clinical Features of Elderly Atrial Fibrillation}

A total of 1144 patients with AF in the Hear Center of our hospital, from September 1st, 2016 to September 30th, 2017, were diagnosed as AF. According to the inclusion and exclusion criteria, 700 elderly patients with AF were enrolled, ranging in age from 65 to 99 -year-old, with an average age of $77.1 \pm 6.9$ years, accounting for $61.2 \%$ of all patients with AF. There were 316 male patients (45.1\%) and 384 female patients $(54.9 \%)$. The ratio of male to female was approximately $1: 1.2$.

\subsubsection{Age and Gender Distribution}

In the elderly population, the proportion of $\mathrm{AF}$ increased with age. The highest proportion of AF occurs in the 7584 age group (49.9\%) (Table 1). According to age, there were more female patients than male patients among different age groups. According to gender, the proportion of 75-84 years old group was the highest in both male and female patients. The chi-square test was performed according to the $\mathrm{X}$-list. There was no significant difference between the groups $(\mathrm{P}<0.05)$.

Table 1. Statistics of age and gender

\begin{tabular}{|c|c|c|c|}
\hline Age & Male & Female & Total \\
\hline $\mathbf{6 5 - 7 4}$ & 110 & 132 & 242 \\
\hline $\mathbf{7 5 - 8 4}$ & 156 & 193 & 349 \\
\hline $\mathbf{8 5}$ and above & 50 & 59 & 109 \\
\hline Total & 316 & 384 & 700 \\
\hline
\end{tabular}

\subsubsection{Relevant etiological statistics}

The causes and related factors of 700 elderly patients with AF were analyzed. As shown in figure 1, 481 cases $(68.7 \%)$ of coronary heart disease, 406 cases $(58.0 \%)$ of hypertension, 341 cases $(48.7 \%)$ of heart failure, 191 cases $(27.3 \%)$ of diabetes, 105 cases $(15.0 \%)$ of valvular disease, 25 cases $(3.6 \%)$ of cardiomyopathy, 21 cases $(3.0 \%)$ of sick sinus syndrome and 20 cases $(2.9 \%)$ of pulmonary heart disease, 15 cases $(2.1 \%)$ of isolated atrial fibrillation and 2 cases $(0.3 \%)$ of congenital heart disease were included. Among them, 41 cases were rheumatic valvular disease, accounting for $39.0 \%$ of valvular disease, accounting for $5.9 \%$ of the total number; 19 cases were senile valvular disease, accounting for $18.1 \%$ of valvular disease, accounting for $2.7 \%$ of the total number.

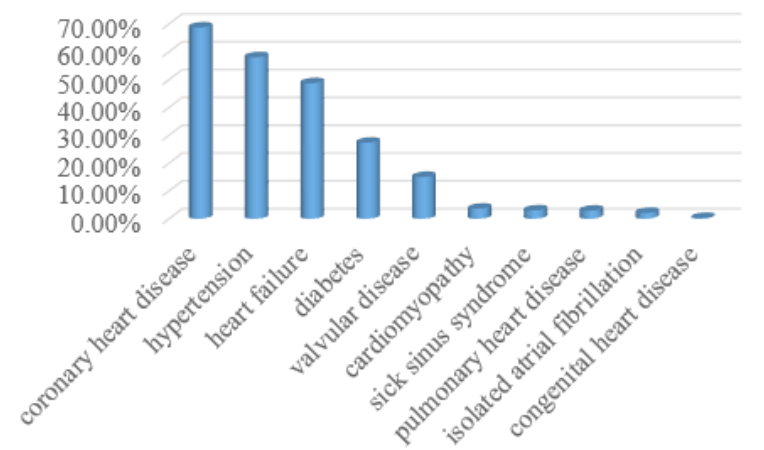

Figure 1. Analysis of the causes and related factors of 700 elderly patients with AF 


\subsubsection{Type distribution of atrial fibrillation}

Among 700 patients with atrial fibrillation, according to the causes, there were 595 patients with non-valvular atrial fibrillation, accounting for $85.0 \%$ of the total number, including 271 male patients and 324 female patients. There were 105 patients with atrial fibrillation, including 45 male patients and 60 female patients. Both types of AF were more common in women; and non-valvular atrial fibrillation was common in both male and female patients. There was no significant difference between them (P > 0.05).

According to the duration of onset, 314 cases of paroxysmal atrial fibrillation, accounting for $44.9 \%$ of the total number, including 134 males and 180 females; 386 cases of persistent atrial fibrillation, accounting for $55.1 \%$ of the total number, including 182 males and 204 females. There was no significant difference between the type of atrial fibrillation and gender composition $(\mathrm{P}>0.05)$.

\subsection{Risk Factors Associated with Persistent Atrial Fibrillation in the Elderly}

\subsubsection{Comparison of Observation Indexes be- tween Paroxysmal Atrial Fibrillation and Per- sistent Atrial Fibrillation}

The general clinical data between the two groups were compared. As shown in Table 2, Patients in the persistent atrial fibrillation group was older than those in the paroxysmal atrial fibrillation group; and the proportion of patients aged $\geq 75$ years was higher. The proportion of patients' persistent atrial fibrillation combined with hypertension, diabetes, coronary heart disease, heart failure, valvular disease and ischemic stroke were higher. Among them, there were significant differences in age, hypertension, heart failure and valvular disease $(\mathrm{P}<0.05)$, and no significant differences in other items $(\mathrm{P}>0.05)$. The two groups of laboratory tests and cardiac color Doppler ultrasound indicators were compared, as shown in Table 3. In the persistent atrial fibrillation group, the levels of CRP, BUA, left atrial inner diameter were higher, and LVEF was lower. There were significant differences in CRP, uric acid, left atrial diameter and LVEF between the two groups $(\mathrm{P}<0.05)$. There was no significant difference in the other indexes between the two groups $(\mathrm{P}>0.05)$.

Table 2: Comparison of general medical records

\begin{tabular}{|c|c|c|c|c|}
\hline & $\begin{array}{c}\text { Paroxysmal atrial } \\
\text { fibrillation } \\
(\mathbf{n}=\mathbf{3 1 4})\end{array}$ & $\begin{array}{c}\text { Persistent atrial } \\
\text { fibrillation } \\
(\mathbf{n}=\mathbf{3 8 6})\end{array}$ & $\begin{array}{c}\text { Statistic } \\
\text { value }\end{array}$ & P value \\
\hline Age & $75.81 \pm 6.58$ & $78.25 \pm 6.96$ & $\mathrm{t}=4.728$ & 0.000 \\
\hline$\geq 75$ years old & 185 & 274 & $x^{2}=11169$ & 0.001 \\
\hline
\end{tabular}

\begin{tabular}{|c|c|c|c|c|}
\hline $\begin{array}{c}\text { Hypertension } \\
\text { (cases) }\end{array}$ & 196 & 210 & $x^{2}=4.568$ & 0.033 \\
\hline $\begin{array}{c}\text { Diabetes (cas- } \\
\text { es) }\end{array}$ & 86 & 105 & $x^{2}=0.003$ & 0.956 \\
\hline $\begin{array}{c}\text { Coronary } \\
\text { heart disease } \\
\text { (cases) }\end{array}$ & 226 & 255 & $x^{2}=2.815$ & 0.093 \\
\hline $\begin{array}{c}\text { Heart failure } \\
\text { (cases) }\end{array}$ & 108 & 233 & $x^{2}=46.734$ & 0.000 \\
\hline $\begin{array}{c}\text { Valvular dis- } \\
\text { ease (cases) }\end{array}$ & 14 & 91 & $x^{2}=49.628$ & 0.000 \\
\hline $\begin{array}{c}\text { Ischemic } \\
\text { stroke (cases) }\end{array}$ & 85 & 99 & $x^{2}=0.181$ & 0.671 \\
\hline
\end{tabular}

Table 3. Comparison of laboratory and cardiac ultrasound indicators

\begin{tabular}{|c|c|c|c|c|}
\hline & $\begin{array}{c}\text { Paroxysmal atrial } \\
\text { fibrillation } \\
(\mathbf{n}=\mathbf{3 1 4})\end{array}$ & $\begin{array}{c}\text { Persistent atrial } \\
\text { fibrillation } \\
(\mathbf{n}=\mathbf{3 8 6})\end{array}$ & Statistic value & P value \\
\hline $\mathbf{C R P}(\mathbf{m g} / \mathbf{L})$ & $815 \pm 2.65$ & $18.15 \pm 5.95$ & $\mathrm{t}=-27.642$ & 0.000 \\
\hline $\mathbf{U A}(\boldsymbol{\mu m o l} / \mathbf{L})$ & $355.06 \pm 94.94$ & $379.26 \pm 108.21$ & $\mathrm{t}=-3.108$ & 0.002 \\
\hline $\begin{array}{c}\text { Creatinine } \\
(\boldsymbol{\mu m o l} / \mathbf{L})\end{array}$ & $98.35 \pm 25.52$ & $99.45 \pm 23.14$ & $\mathrm{t}=-0.596$ & 0.551 \\
\hline TC (mmol/L) & $3.93 \pm 0.92$ & $3.91 \pm 0.89$ & $\mathrm{t}=0.386$ & 0.699 \\
\hline TG (mmol/L) & $1.19 \pm 0.34$ & $1.24 \pm 0.39$ & $\mathrm{t}=-1.572$ & 0.116 \\
\hline $\mathbf{L D L}(\mathbf{m m o l} / \mathbf{L})$ & $2.38 \pm 0.59$ & $2.41 \pm 0.85$ & $\mathrm{t}=-0.486$ & 0.627 \\
\hline $\begin{array}{c}\text { Left atrial } \\
\text { inner diameter } \\
(\mathbf{m m})\end{array}$ & $37.54 \pm 5.40$ & $51.01 \pm 7.71$ & $\mathrm{t}=-5.053$ & 0.000 \\
\hline $\mathbf{L V E F}(\%)$ & $53.68 \pm 7.04$ & $47.53 \pm 4.75$ & $\mathrm{t}=-13.734$ & 0.000 \\
\hline
\end{tabular}

\subsubsection{Spearman Correlation Analysis}

Spearman correlation analysis was performed on the above eight statistically significant items, as shown in Table 4 . The results showed that persistent atrial fibrillation was positively correlated with age, heart failure, valvular disease, CRP, uric acid, and left atrial diameter; and negatively correlated with LVEF, but not with hypertension.

Table 4. Spearman correlation analysis

\begin{tabular}{|c|c|c|}
\hline & r value & P value \\
\hline Age & 0.181 & 0.000 \\
\hline Hypertension & -0.055 & 0.146 \\
\hline Heart failure & 0.262 & 0.000 \\
\hline Valvular disease & 0.266 & 0.000 \\
\hline CRP & 0.820 & 0.000 \\
\hline Uric acid & 0.166 & 0.000 \\
\hline Left atrial diameter & 0.167 & 0.000 \\
\hline LVEF & -0.477 & 0.000 \\
\hline
\end{tabular}

\subsubsection{Multivariate Logistic Regression Analysis}

Multivariate logistic regression analysis was performed on relevant 7 items including age, CRP, uric acid, left 
atrial diameter, LVEF, heart failure, and valvular disease. The results showed that CRP level and left atrial inner diameter were independent risk factors for persistent atrial fibrillation in the elderly.

\section{Discussion}

\subsection{Clinical Characteristics of Atrial Fibrillation in the Elderly}

\subsubsection{Age}

Many studies have confirmed that age is an independent risk factor for atrial fibrillation, and is now widely recognized. Patients with atrial fibrillation aged 65 and over are specifically referred to as atrial fibrillation in the elderly ${ }^{[10]}$. The incidence of AF increases with age. The Framingham study showed that the incidence of AF was $0.5 \%$ in the $50-59$ age group, $1.8 \%$ in the $60-69$ age group, $4.8 \%$ in the $70-79$ age group, and $8.8 \%$ in the 80 89 age group. The incidence of AF increased significantly after 60 years old, with an average annual increase of 1 fold ${ }^{[6]}$. According to a study published by the EHRA (European Heart Rhythm Association), the prevalence of AF in people younger than 60 years is about $1 \%$; the prevalence of people aged $75-84$ years is increased to $12 \%$, and more than $1 / 3$ after 80 years old ${ }^{[11]}$. The expert consensus of FSGG/FSC (French Society of Gerontology and Geriatrics/French Society of Cardiology) in 2013 pointed out that $70 \%$ of patients with $\mathrm{AF}$ are older than or equal to 75 years old ${ }^{[12]}$. At present, there is a lack of large-scale atrial fibrillation data statistics in China. In 2004, Zhou et al. carried out a large-scale epidemiological study on 29079 cases of 14 natural populations. Among them, the prevalence rate was $0.5 \%$ in the $50-59$ age group, and $7.5 \%$ in the 80 -year-old group ${ }^{[7]}$.

The results of this study showed that in elderly patients with AF, patients over 75 years of age were the main patient group. This is consistent with the above findings. Patients aged 75-84 accounted for the majority of patients. Compared with that, the proportion of patients over 85 years of age did not increase significantly, which may be related to the small number of elderly hospitalized, population, regional differences and other factors.

The prevalence rate of AF increases with age; and it occurs more frequently in the elderly, which is associated with a series of body changes caused by aging. It has been suggested in the literature that when the human body enters the aging stage and develops further with the aging degree, the expression of L-type calcium channels and contractile proteins decreases at the gene and protein levels, and the left atrium (LA) may become more prominent in the occurrence and development of AF. The specific reasons and possible mechanisms are not fully known and determined yet, which needs to be further studied ${ }^{[13]}$.

\subsubsection{Gender}

The 38-year follow-up study of Framingham ${ }^{[6]}$ in the United States showed that the prevalence of AF in men was 1-5 times higher than that in women. Studies in China have shown that the incidence of male AF is slightly higher than that of females ${ }^{[7,14]}$. But other studies have suggested further points. Feinberg et al. showed that male patients are more common than female patients before the age of 75; and after 75 years old, the proportion of female patients with atrial fibrillation increased, which was about $60 \%{ }^{[15]}$. Other studies have shown that the prevalence of women in the age group $>75$ years old is twice that of men ${ }^{[16]}$. According to statistics from Switzerland, the prevalence of atrial fibrillation among women under 80 is lower than that of men, and women over 80 are higher than men ${ }^{[17]}$. In 2015 , a study showed that the incidence of atrial fibrillation in China was $0.05 / 100$ patients · year. After age adjustment, the incidence and prevalence of atrial fibrillation in women were lower than in men, but the prevalence of atrial fibrillation in elderly women increased significantly ${ }^{[18]}$. This suggests that gender differences in atrial fibrillation may vary with age. The results of this study indicate that the prevalence of atrial fibrillation in older women is higher than that in men. The male to female ratio is about 1:1.2, and the prevalence of women in all age groups is high, which is roughly consistent with the above findings. The higher prevalence of atrial fibrillation in older women may be associated with changes in estrogen levels, autonomic dysfunction, increased risk factors for comorbidity in older women, and longer life expectancy in women. The specific reasons still need further discussion. Patients with atrial fibrillation have a faster ventricular rate and more pronounced symptoms. Compared with men, the quality of life is lower, and the mortality rate of female atrial fibrillation is not lower than that of men ${ }^{[19-21]}$.

\subsection{Risk Factors Associated with Persistent Atrial Fibrillation}

\subsubsection{Atrial Fibrillation and Heart Failure}

In the American Flemingham study, the annual incidence of atrial fibrillation in heart failure patients was $54 \%$, and the annual incidence of central dysfunction in patients with atrial fibrillation was $33 \%{ }^{[22]}$. Atrial fibrillation interacts with heart failure. They have common risk factors such as age, smoking, obesity, hypertension, diabetes, 
renal impairment, valvular heart disease, coronary artery disease, and obstructive sleep apnea. Pathophysiological mechanisms, neuroendocrine, electrophysiological and hemodynamic changes promote each other and form a vicious cycle ${ }^{[23]}$. Diastolic heart failure is common in the elderly population, and the incidence of arrhythmia is significantly higher than that of systolic heart failure. The results of this study suggest that heart failure is closely associated with persistent atrial fibrillation in the elderly. Increased volume load in patients with heart failure can lead to atrial dilatation, atrial stretch can activate ion channels, abnormal cardiac conduction, shortened refractory period and other changes, and increased blood volume can activate RASS system, which is conducive to the maintenance of atrial fibrillation. Meanwhile, the application of digoxin and other drugs has a certain impact on the progress of atrial fibrillation. In the later stage, the effects of heart function grading and anti-heart failure medication on persistent atrial fibrillation can be further studied.

\subsubsection{Atrial Fibrillation and Hypertension}

Hypertension is an independent predictor of newly onset atrial fibrillation and is almost linearly correlated with the occurrence of atrial fibrillation. Meanwhile, multiple studies have confirmed that prehypertension is also a high-risk factor related to atrial fibrillation ${ }^{[24-26]}$. Most old people have arteriosclerosis, and the difference in pulse pressure is large. Foreign studies suggest that an increase of $20 \mathrm{mmHg}$ in pulse pressure can increase the risk of atrial fibrillation ${ }^{[27]}$. Studies on elderly patients with hypertension have found a certain correlation between non-arytenoid blood pressure and the occurrence of atrial fibrillation ${ }^{[28]}$. It is suggested that reducing blood pressure variability in hypertensive patients can reduce the recurrence of atrial fibrillation and related target organ damage. Meanwhile, other scholars have put forward different conclusions. A meta-analysis conducted by Webb et al. pointed out that the blood pressure variability in patients with hypertension did not reduce the risk of new atrial fibrillation ${ }^{[29]}$. Blood pressure variability can be used as a predictor of risk, but may not be suitable as a therapeutic target. Whether it is an independent risk factor for atrial fibrillation needs further study. In this study, hypertension was associated with persistent atrial fibrillation. The left atrial structure changes induced by chronic hypertension provide conditions for the maintenance of atrial fibrillation. The vascular endothelium is damaged by hypertension, which further leads to atherosclerosis. Increased arterial stiffness is characterized by increased pulse differential pressure, which increases the risk of atrial fibrillation. In this study, the relationship between hypertension and atrial fibrillation was not further studied in terms of grading of hypertension and pulse pressure, which is the deficiency of this study.

\section{Conclusion}

(1) In elderly patients with atrial fibrillation, the proportion of atrial fibrillation increases with age. The age group between 75 and 84 years old accounted for the largest proportion, and the proportion of elderly women patients was higher, and slightly higher than that of men in all age groups. Elderly women with atrial fibrillation need to be taken seriously.

(2) Non-valvular atrial fibrillation is most common in elderly patients with atrial fibrillation. The most common first three related causes or risk factors are coronary heart disease, hypertension, and heart failure. The comorbidity of elderly patients with atrial fibrillation is common, and comorbid management is the prevention and treatment target of atrial fibrillation in the elderly.

\section{Reference}

[1] Chugh S S, Havmoeller R Narayanan K, et a1. Worldwide Epidemiology of Atrial Fibrillation: A Global Burden of Disease 2010 Study[J]. Circulation, 2014, 129(8): 837-847.

[2] Miyasaka Y, Barnes M E, Gersh B J, et al. Secular trends in incidence of atrial fibrillation in OImsted County, Minnesota, 1980 to 2000, and implications on the projections for future prevalence $[\mathrm{J}]$. Circulation, 2006, 114(2): 119-125.

[3] Go A S, Hylek E M, Phillips K A, et a1. Prevalence of diagnosed atrial fibrillation in adults: national implications for rhythm management and stroke prevention: The Anticoagulation and Risk Factors in Atrial Fibrillation (ATRIA)Study [J]. Jama, 2001, 285(18): 2370.

[4] Krijthe B P, Kunst A, Benjamin E J, et a1. Projections on the number of individuals with atrial fibrillation in the European Union, from 2000 to 2060[J]. European Heart Journal, 2013, 34(35): 2746-51.

[5] Chiang C E, Wang K L, Lip G Y. Stroke prevention in atrial fibrillation: an Asian perspective[J]. Thromb Haemost, 2014, 111(5): 789-97.

[6] Kannel W B, Abbott R D, Savage D D, et a1. Epidemiologic features of chronic atrial fibrillation: the Framingham study [J]. New England Journal of Medicine, 1982, 306(17): 1018.

[7] Ziqiang Zhou, Dayi Hu, Jie Chen, et al. Epidemiological study on the current status of atrial fibrillation in China [J]. Chinese Journal of Internal Medicine, 2004, 43(7): 491-494. 
[8] Stewart S, Hart C L, Hole D J, et al. A population-based study of the long-term risks associated with atrial fibrillation: 20-year follow-up of the Renfrew/Paisley study[J]. American Journal of Medicine, 2002, 113(5): 359-364.

[9] Rich M W, Chyun D A, Skolnick A H, et a1. Knowledge Gaps in Cardiovascular Care of the Older Adult Population: A Scientific Statement from the American Heart Association, American College of Cardiology, and American Geriatrics Society[J]. Journal of the American College of Cardiology, 2016, 67(20): 2419-2440.

[10] Chinese Experts'Suggestions on the Diagnosis and Treatment of Atrial Fibrillation in the Elderly (2011) $[\mathrm{J}]$. Chinese Journal of Practical Internal Medicine, 2012, 30(4): 894-908.

[11] Carom A J, Kirchhof P, Lip G Y H, et al. Guidelines for the management of atrial fibrillation The Task Force for the Management of Atrial F1brillation of the European Society of Cardiology (ESC)[M]//European Heart Journal. 2010: 2369-2429.

[12] Hanon O, Assayag P, Belmin J, et a1. Expert consensus of the French Society of geriatrics and gerontology and the French society of cardiology on the management of atrial fibrillation in elderly people [J]. Archives of Cardiovascular Diseases, 2013, 106(5): 303-23.

[13] Chinese Society of Cardiovascular Diseases of Chinese Medical Association. Retrospective investigation of hospitalized cases of atrial fibrillation in parts of China [J]. Chinese Journal of Cardiology, 2003, 31(12): 913-916.

[14] Yanjun Gong, Wenhui Ding, Yansheng Ding, et al. Analysis of etiology (or related factors) in 1335 patients with atrial fibrillation [J]. Chinese Journal of Geriatric Heart Brain and Vessel Diseases. 2006, 8(10): 658-661.

[15] Feinberg W M, Blackshear J L, Laupacis A, et al. Prevalence, age distribution gender of patients with atrial fibrillation. Analysis and implications [J]. Archives of Internal Medicine, 1995, 155(5): 469.

[16] Fang M C, Singer D E, Chang Y et a1. Gender differences in the risk of ischemic stroke and peripheral embolism in atrial fibrillation: The AnTicoagulation and Risk factors In Atrial fibrillation (ATRIA)study [J]. Circulation, 2005, 112(12): 1687-1691.

[17] Andersson T, Magnuson A, Bryngelsson I L, et a1. All-cause mortality in 272186 patients hospitalized with incident atrial fibrillation 1995 2008: a Swedish nationwide long-term case-control study[J]. European Heart Journal, 2013, 34(14): 1061.

[18] Guo Y, Tian Y, Wang H, et a1. Prevalence, incidence, and lifetime risk of atrial fibrillation in China: new insights into the global burden of atrial fibrillation[J]. Chest, 2015, 147(1): 109-119.

[19] Chugh S S, Havmoeller R, Narayanan K, et al. Worldwide Epidemiology of Atrial Fibrillation: A Global Burden of Disease 2010 Study [J]. Circulation, 2014, 129(8): 837-47.

[20] Ko D, Rahman F, Schnabel R B, et a1. Atrial fibrillation in women: epidemiology, pathophysiology, presentation and prognosis[J]. Nature Reviews Cardiology, 2016, 13(6): 321.

[21] Ning Wang, Mingzhao Qin, Current status of treatment of female nonvalvular atrial fibrillation $[\mathrm{J}]$. Chinese Journal of Geriatric Heart Brain and Vessel Diseases, 2018(1): 99-101.

[22] Chinese expert consensus on diagnosis and treatment of chronic heart failure combined with atrial fibrillation. Chinese Journal of Medicine, 2011, 46: 78-84.

[23] Wentao Ma, Xiaohan Fan, Shu Zhang, Therapeutic development direction of atrial fibrillation complicated with heart failure [J]. Chinese Circulation Journal, 2016, 31(11): 1142-1144.

[24] O'Neal W T, Soliman E Z, Qureshi W, et a1. Sustained pre-hypertensive blood pressure and incident atrial fibrillation: The Multi-Ethnic Study of Atherosclerosis[J]. Journal of the American Society of Hypertension Jash, 2015, 9(3): 191-196.

[25] Cohen D, Tedrow UB, Koplan BA, et a1. Influence of systolic and diastolic blood pressure on the risk of incident atrial fibrillation in women. Circulation 2009; 119: 2146-52.

[26] Grundvold I, Skretteberg PT, LiestM K, et a1. Upper normal blood pressures predict incident atrial fibrillation in healthy middle-aged men: a 35-year follow-up study. Hypertension 2012; 59: 198-204.

[27] Michell GF, Vasan RS, Keyes MJ, et a1. Pulse pressure and risk of new-onset atrial fibrillation. JAMA 2007; 297: 709-15.

[28] Wenqing Lei, Correlation between circadian rhythm of blood pressure and paroxysmal atrial fibrillation in elderly hypertensive patients [J]. Chinese Journal of Geriatric Care, 2016, 14(1): 16-20.

[29] Webb AJ, Rothwell PM. Blood pressure variability and risk of new-onset atrial fibrillation: a systematic review of randomized trials of antihypertensive drugs [J]. Stroke, 2010, 41(9): 2091-2093. 